\title{
A High Order Accurate Nodal Discontinuous Galerkin Method (DGM) for Numerical Solution of Hyperbolic Equation
}

\author{
Fareed Ahmed, Faheem Ahmed,Yonghang Guo, and Yong Yang
}

\begin{abstract}
This paper deals with a high-order accurate Nodal Discontinuous Galerkin (DG) method for the numerical solution of the inviscid Burgers equation, which is a simplest case of nonlinear, hyperbolic partial differential equation. This method combines mainly two key ideas which are based on the finite volume and finite element methods. The physics of wave propagation being accounted for by means of Riemann problems and accuracy is obtained by means of high-order polynomial approximations within elements. In Nodal DG method a finite element space discretization is obtained by element wise discontinuous approximations. Whereas low-storage, high order accurate, explicit Runge-Kutta (LSERK) method is used for temporal discretization. The resulting RKDG methods are stable, high-order accurate and highly parallelizable schemes that can easily handle complicated geometries and boundary conditions. Exponential filter is used to remove spurious oscillations near the shock waves. The $L_{2}$ and $L_{\infty}$ errorsin the solution show that the scheme is accurate and effective. Hence, the method is well suited to achieve high order accurate solution for the hyperbolic partial differential equations.
\end{abstract}

Index Terms-Nodal Discontinuous galerkin method, burgers equation, exponential filter, hyperbolic PDE.

\section{INTRODUCTION}

The Discontinuous Galerkin Method (DGM) was first introduced by Reed and Hill [1] as a technique to solve neutron transport problems. In a series of papers by Cockburn, Shu et al. [2-5], the RKDG method has been developed for solving nonlinear hyperbolic conservation laws and related equations, in which DG is used for spatial discretization with flux values at cell edges computed by either Riemann solversor monotone flux functions, the total variation bounded (TVB) limiter [6-7] is employedto eliminate spurious oscillations and the total variation diminishing (TVD) Runge-Kutta(RK) method is used for the temporal discretization to ensure the stability of the numericalapproach while simplifying the implementation.

The Discontinuous Galerkin Method (DGM) has recently become more popular for the solution of systems of conservation laws to arbitrary order of accuracy [8], [9]. An intelligent combination of the finite element and finite volume method, utilizing a space of basis and test function that mimics thefinite element method but satisfying the

Manuscript received May 18, 2012; revised June 12, 2012

The authors are with the Northwestern Polytechnical University, Xi' an, China (Tel.: +8618629067124; e-mail: fareedmuet@yahoo.com). equation in a sense closer to the finite volume method, appears to offer many of the desired properties. This combination is exactly what leads to Discontinuous Galerkin Finite Element Method (DG-FEM) [10]. The physics of wave propagation is, however, accounted for by solving the Riemann problems that arise from the discontinuous representation of the solution at element interfaces [11], [12], [13].

High order accurate Low-Storage Explicit Runge-Kutta (LSERK) method is used for temporaldiscretization.The resultingRKDG methods are stable, high-order accurate and highly parallelizable schemesthat can easily handle complicated geometries and boundary conditions [14]. Many of the equations of mechanics are hyperbolic, and so the study of hyperbolic equations is of substantial contemporary interest.

\section{NODAL DiscontinUOUS GALERKIN FORMULATION}

\section{A. Spatial Discretization}

The conservation law is discretized in space by using Discontinuous Galerkin approach. Here we consider problem posed on the physical domain $\Omega$ with boundary $\partial \Omega$ and assume that this domain is well approximated by the computational domain $\Omega_{h}$. This is space filling triangulation composed of a collection of $\mathrm{k}$ geometry-conforming non-overlapping elements $\mathrm{D}^{\mathrm{k}}$. The model equation is

$$
\frac{\partial u}{\partial t}+\frac{\partial f(u)}{\partial x}=0, \quad x \quad \in[L, R]
$$

where the flux is given as $f(u)=\frac{u^{2}}{2}$.It is subject to the initial conditions and boundary conditions of the form:

$$
\begin{gathered}
u(x, 0)=u_{0}(x)=-x, \quad u(L, t)=u(-1, t)=1, \\
u(R, t)=u(1, t)=-1
\end{gathered}
$$

We approximate $\Omega$ by $K$ non-overlapping elements, $x \in\left[x_{l}^{k}, x_{r}^{k}\right]=D^{k}$, as illustrated in Fig. 1. On each of these elements we express the local solution as a polynomial of order $N=N_{p}-1$

$$
u_{h}^{k}(x, t)=\sum_{i=1}^{N_{p}} u_{h}^{k}\left(x_{i}^{k}, t\right) l_{i}^{k}(x)=\sum_{i=1}^{N_{p}} u_{h i}^{k}(t) l_{i}^{k}(x)
$$




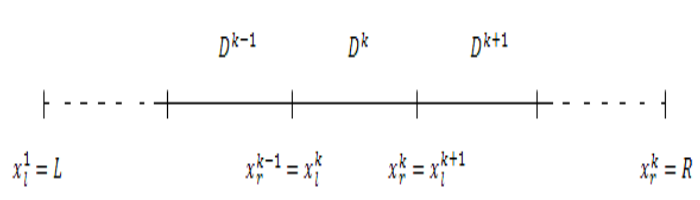

Fig. 1. Computational domain

In Eq. (2) we have applied nodal approach where we introduce $N_{p}=N+1$ local grid points, $x_{i}^{k} \in D^{k}$, and express the polynomial through the associated interpolating Lagrange Polynomial, $l_{i}^{k}(x)$. The global solution is assumed to be approximated by the piecewise $\mathrm{N}$-th order polynomial approximation $u_{h}(x, t)$, defined as the direct sum of the $K$ local polynomial solution $u_{h}^{k}(x, t)$ as

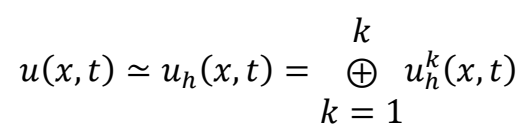

The inner product of residual and basis must be zero

$$
\int_{x^{k}}^{x^{k+1}} R_{h}(x, t) l_{j}^{k}(x) d x=0
$$

Introducing affine mapping

$$
x \in D^{k}: x(r)=\frac{x^{k}+x^{k+1}}{2}+\frac{x^{k+1}-x^{k}}{2} r .
$$

The reference variable $r \in[-1,1]$.

Using Gauss' theorem to obtain local statement

$$
\int_{D^{k}} \frac{\partial u_{h}^{k}}{\partial t} l_{j}^{k}-f_{h}^{k} \frac{\partial l_{j}^{k}}{\partial x} d x=-\left[f_{h}^{k} l_{j}^{k}\right]_{x^{k}}^{x^{k+1}}
$$

The main purpose of the right hand side is to connect the elements. Further, considering the local solution as approximation to the global solution yields the local semi-discrete scheme as

$$
M^{k} \frac{d u_{h}^{k}}{d t}-\left(S^{k}\right)^{T} f_{h}^{k}=-\left[f_{h}^{k} l_{j}^{k}\right]_{x^{k}}^{x^{k+1}}
$$

where $M^{k}$ and $S^{k}$ are mass and stiffness matrices of element krespectively and computed as under

$$
\begin{array}{r}
M_{i j}^{k}=\left(l_{i}^{k}, l_{j}^{k}\right)_{D^{k}}=\int_{x^{k}}^{x^{k+1}} l_{i}^{k}(x) l_{j}^{k}(x) d x, \\
S_{i j}^{k}=\int_{D^{k}} l_{i}^{k}(x) \frac{d l_{j}}{d x} d x
\end{array}
$$

Finally, Eq. (5) can be written in the matrix form as

The numerical flux $f^{*}$ is calculated by using monotone Lax-Friedrich scheme. Here, the concept of approximate Riemann solver or numerical flux is incorporated into the DG method.

$$
\begin{gathered}
\frac{h^{k}}{2}[M]_{n \times n} \frac{d}{d t}\left[\begin{array}{c}
u_{h 1}^{k} \\
u_{h 2}^{k} \\
\vdots \\
u_{h n}^{k}
\end{array}\right]-[S]_{n \times n}^{T}\left[\begin{array}{c}
f_{h 1}^{k} \\
f_{h 2}^{k} \\
\vdots \\
f_{h n}^{k}
\end{array}\right] \\
=-f_{h}^{*}\left(x^{k+1}\right)\left[\begin{array}{c}
0 \\
0 \\
0 \\
\vdots \\
0 \\
1
\end{array}\right]+f_{h}^{*}\left(x^{k}\right)\left[\begin{array}{c}
1 \\
0 \\
0 \\
\vdots \\
0
\end{array}\right]
\end{gathered}
$$

\section{B. Time Discretization}

Subsequent to space discretization, the resulting system of ordinary differential equations, $\frac{d u_{h}}{d t}=L\left(u_{h}, t\right)$, is discretized by using explicit high-order accurate Low-Storage Explicit Runge-Kutta (LSERK) method:

1) $\operatorname{Set} p^{(0)}=u^{n}$

2) Compute the intermediate functions:

$$
i \in[1, \ldots, 5]\left\{\begin{array}{c}
k^{(i)}=\alpha_{i} k^{(i-1)}+\Delta t L_{h}\left(p^{(i-1)}, t^{n}+\gamma_{i} \Delta t\right) \\
p^{(i)}=p^{(i-1)}+\beta_{i} k^{(i)}
\end{array}\right.
$$

3) Set $u_{h}^{i+1}=p^{(5)}$

where $\alpha, \beta$ and $\gamma$ are the coefficients needed in the LSERK.

\section{FILTERING}

In order to curb spurious oscillations in vicinity of shock wave an exponential filter is used. Filter matrix F defined as

$$
F=V \Lambda V^{-1}
$$

where $\mathrm{F}$ is filter matrix and $\Lambda$ is the diagonal matrix having entries

$$
\Lambda_{i i}=\sigma\left(\frac{i-1}{N}\right), \quad i=1, \ldots, N p
$$

$$
=\left\{\begin{array}{cc}
\boldsymbol{1}(\boldsymbol{\eta}) & 0 \leq \boldsymbol{\eta} \leq \boldsymbol{\eta}_{\boldsymbol{c}} \\
\exp \left(-\alpha\left(\frac{\eta-\eta_{c}}{1-\eta_{c}}\right)^{s}\right), & \boldsymbol{\eta}_{c} \leq \boldsymbol{\eta} \leq 1
\end{array}\right\}
$$

where $\eta_{c}=\frac{N c}{N}, N c$ represents a cutoff below which the low modes are left untouched.

\section{NUMERICAL RESULTS}

Similar to other Finite Element Methods, complex geometries and boundary conditions can easily be handled with nodal DG method. High-order accuracy can also be attained. The results obtained are depicted in the following Figs. 


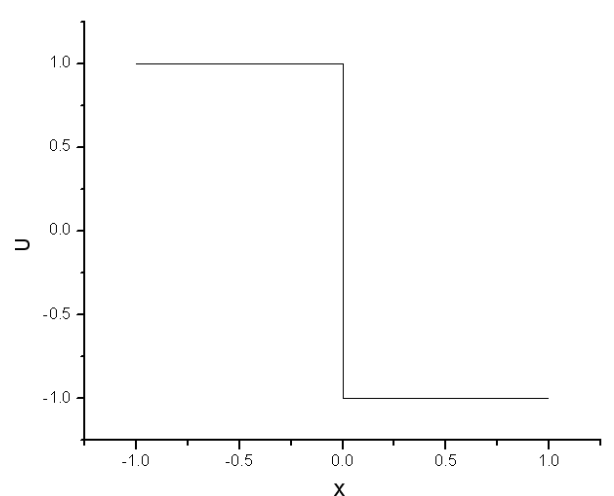

Fig. 2. Exact solution.

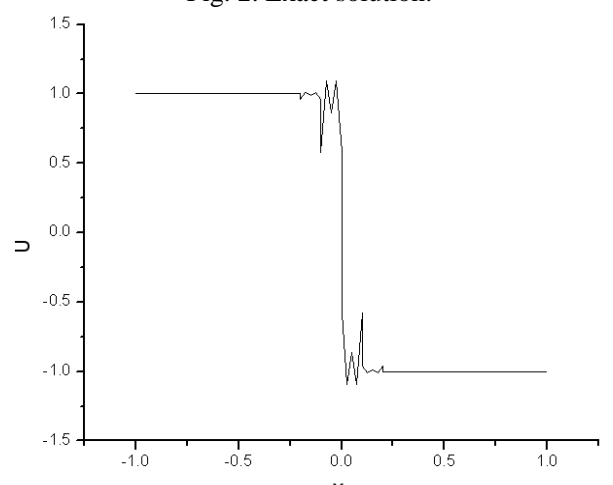

Fig. 3. Numerical solution before filtering.

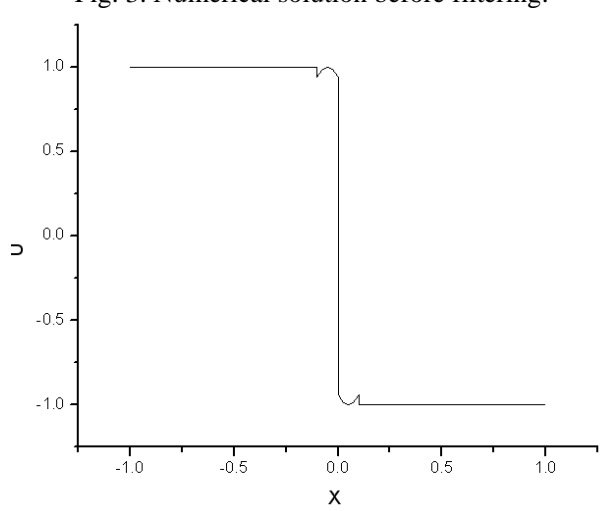

Fig. 4. Numerical solution after filtering.

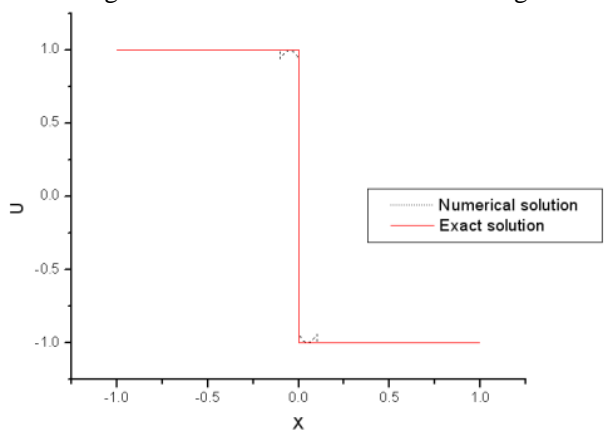

Fig. 5. Comparison of computed result with exact solution.

The $L_{2}$ and $L_{\infty}$ error norms are used to measure the accuracy of scheme as shown in Table I.

\section{CONCLUSION}

The numerical solution of inviscid Burgers Equation, obtained by using Nodal DGM is highly accurate and nearly the same as exact solution as shown in Fig. 5. In order to remove wiggles as shown in Fig. 3, a filter was constructed and added in the solution, due to which the wiggles near the shock wave were considerably removed as shown in Fig.4 and Fig. 5. From table I, it can be observed that with decreasing the step size, the errors in the solution also decrease. However, different types of filters and limiters can be constructed to further smoothen the solution near the shockwave

TABLE I: $L_{2}$ AND $L_{\infty}$ ERRORS AT DIFFERENT STEP SIZES AND POLYNOMIAL ORDER.

\begin{tabular}{|c|c|c|c|c|}
\hline \multirow{2}{*}{$h$} & \multicolumn{3}{|c|}{$p^{3}$} & \multicolumn{2}{c|}{$p^{4}$} \\
\cline { 2 - 5 } 20 & $L_{2}$ & $L_{\infty}$ & $L_{2}$ & $L_{\infty}$ \\
\hline \multirow{2}{*}{30} & $1.66 \mathrm{E}-01$ & $8.23 \mathrm{E}-02$ & $1.20 \mathrm{E}-01$ & $6.77 \mathrm{E}-02$ \\
\hline 40 & $1.27 \mathrm{E}-01$ & $6.89 \mathrm{E}-02$ & $1.06 \mathrm{E}-01$ & $5.91 \mathrm{E}-02$ \\
\hline 50 & $1.08 \mathrm{E}-01$ & $6.14 \mathrm{E}-02$ & $9.27 \mathrm{E}-02$ & $4.86 \mathrm{E}-02$ \\
\hline
\end{tabular}

\section{REFERENCES}

[1] Reed and T. Hill. "Triangular mesh methods for the neutron transport equation," Tech. report la-ur-73-479, Los Alamos Scientific Laboratory, 1973.

[2] B. Cockburn and C.-W. Shu, "The TVB Runge-Kutta local projection discontinuous Galerkin finite element method for conservation laws V: multidimensional systems," J. Comput. Phys., vol. 141, pp. 199-224, 1998.

[3] B. Cockburn, S. Hou, and C.-W. Shu, "The TVB Runge-Kutta local projection discontinuous Galerkin finite element method for conservation laws IV: the multidimensional case," Math. Comp., vol. 54, pp. 545-581, 1990

[4] B. Cockburn, S.-Y. Lin, and C.-W. Shu, "TVB Runge-Kutta local projection discontinuous Galerkin finite element method for conservation laws III: one dimensional systems," J. Comput. Phys., vol. 52, pp. 411-435, 1989 .

[5] B. Cockburn and C.-W. Shu, "TVB Runge-Kutta local projection discontinuous Galerkin finite element method for conservation laws II: general framework," Math. Comp., vol. 52, pp. 411-435, 1989.

[6] C.-W. Shu and S. Osher, "Efficient implementation of essentially non-oscillatory shock capturing schemes II," J. Comput. Phys., vol. 83 , pp. 32-78, 1989

[7] P. Woodward and P. Colella, "Numerical simulation of two-dimensional fluid flows with strong shocks," J. Comput. Phys., vol. 54, pp. 115, 1984.

[8] H. Luo, J. D. Baum, and R. Lohner, "Discontinuous galerkin method based on a taylor basis for the compressible flows on arbitrary grids," Journal of Computational Physics, 2008.

[9] H. Luo, J. D. Baum, and R. L"ohner, "On the computation of steady-state compressible flows using a discontinuous galerkin method," International Journal for Numerical Methods in Engineering, vol. 73 , no. 5 , pp. 597-623, 2008

[10] J. S. Hesthaven and T. Warburton, "Nodal discontinuous galerkin methods- algorithms, analysis, and applications," 2008.

[11] F. Bassi and S. Rebay, "A high-order accurate discontinuous finite element method for the numerical solution of the compressible navier-stokes equations," Journal of Computational Physics, vol. 131, pp. 267-279, 1997.

[12] H. Luo, J. D. Baum, and R. L"ohner, "A p-multigrid discontinuous galerkin method for the euler equations on unstructured grids," Journal of Computational Physics, vol. 211, no. 2, pp. 767-783, 2006.

[13] H. Luo, J. D. Baum, and R. L"ohner, "Fast, p-multigrid Discontinuous Galerkin Method for compressible flows at All Speeds," AIAA Journal, vol. 46, no. 3, pp. 635-652, March 2008.

[14] B. Cockburn and C.-W. Shu, "Runge - kutta discontinuous galerkin methods for convection-dominated problems," Journal of Scientific Computing, vol. 16, no. 3, September 2001 